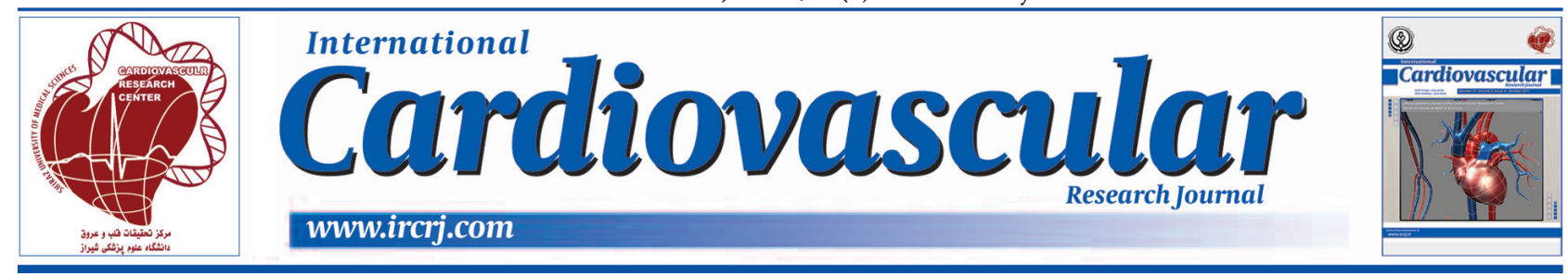

\title{
Cytomegalovirus Infection and Coronary Artery Disease: A Single- Center Serological Study in Northwestern Iran
}

\author{
Zakieh Rostamzadeh Khameneh ${ }^{1}$, Alireza Rostamzadeh ${ }^{2}$, Mohaddeseh Nemati ${ }^{3,4}$, Paul M. Brown ${ }^{5}$, \\ Nariman Sepehrvand ${ }^{4,5, *}$
}

${ }^{1}$ Department of Microbiology and Immunology, Urmia University of Medical Sciences, Urmia, IR Iran

${ }^{2}$ Department of Cardiology, Urmia University of Medical Sciences, Urmia, IR Iran

${ }^{3}$ Department of Biochemistry, Urmia University of Medical Sciences, Urmia, IR Iran

${ }^{4}$ Students' Research Committee, Urmia University of Medical Sciences, Urmia, IR Iran

${ }^{5}$ Department of Medicine, University of Alberta, Edmonton, Alberta, Canada

\begin{tabular}{l}
\hline A R T I C L E I N F O \\
\hline Article Type: \\
Research Article \\
\hline Article History: \\
Received: 07 Aug 2015 \\
Revised: 19 Jan 2016 \\
Accepted: 29 Feb 2016 \\
\hline
\end{tabular}

Keywords:

Cytomegalovirus

Coronary Artery Disease

Coronary Angiography

\begin{abstract}
A B S T R A C T
Background: The role of chronic Cytomegalovirus (CMV) infection and inflammation in the pathogenesis of atherosclerosis and Coronary Artery Disease (CAD) is still not clear. Objectives: This study aimed to explore the seroprevalence of anti-CMV antibodies and inflammatory markers in patients with stable angina who had undergone diagnostic coronary angiography for clinical suspicion of CAD.

Patients and Methods: This cross-sectional, descriptive study was conducted on 181 patients with stable angina selected randomly among the patients referred to Seyyedoshohada Heart Hospital of Urmia, Iran for diagnostic coronary angiography between August 2012 and December 2013. The patients were categorized into CAD and non-CAD groups based on their angiographic findings. Then, anti-CMV IgG and IgM antibodies were tested using the Enzyme-Linked Immunosorbent Assay (ELISA) method (Diapron, Rome, Italy). Serum C-Reactive Protein (CRP) was also measured by a qualitative method (Aniston Kit).

Results: Based on angiographic findings, 141 patients (77.9\%) had atheromatous plaques in their coronary arteries, while coronary arteries were free of any plaques in 40 cases (22.1\%). Besides, $99.3 \%$ of the patients in the CAD group and all the patients in the non-CAD group were anti-CMV IgG positive. The rate of anti-CMV IgM seropositivity was $11.7 \%$ in the CAD group and $13.2 \%$ in the non-CAD group $(\mathrm{P}=0.78)$. However, no significant difference was observed between the groups with and without angiographicallydocumented CAD in terms of CRP seropositivity ( $64.7 \%$ vs. $56.4 \%, \mathrm{P}=0.34)$.

Conclusions: Regardless of having angiographically-proven CAD, almost all the cases referred for coronary angiography in our study had a previous exposure to CMV infection as determined by the presence of anti-CMV IgG antibodies in their sera. In fact, the results indicated no significant associations between CMV infection and the presence of CAD.
\end{abstract}

Implication for health policy/practice/research/medical education:

The results of the current study showed that because of widespread exposure of Iranian general population to CMV infection, almost all the cases who referred for coronary angiography in our hospital were seropositive for CMV. However, no association was observed between CMV infection and the presence of CAD, which could be justified by the high rate of CMV-specific Ig G seropositivity. This finding rejects the need for and benefit of serological assessment of patients with suspected acute coronary syndrome for CMV in Iran.

\section{Background}

Cytomegalovirus (CMV) is a $\beta$-herpesvirus, which infects

*Corresponding author: Nariman Sepehrvand, Students' Research Committee, Deputy for Research Affairs, Urmia University of Medical Sciences, Resalat Ave. Djahad Square, Urmia, West-Azerbaijan, Iran. Cellphone: +98-9125936372, E-mail:nariman256@gmail.com.
$40-100 \%$ of adults and is endemic in all regions of the world $(1,2)$. It could be associated with persistent, latent, and recurrent infections. Besides, it has been shown that the reactivations of the latent virus could happen in the vascular wall cells (3).

Despite a decade of experimental and clinical 
investigations, the role of inflammation and infections in the pathogenesis of atherosclerosis is still not clear. Among all other pathogens, CMV infection was studied more and was claimed to be a potential trigger for atherosclerosis and Coronary Artery Disease (CAD) (4). However, the available data are not entirely consistent. Some studies showed a significant association between CMV infection and atherosclerosis/CAD (3, 5-10), while some others did not (11-23). Ji et al. conducted an aggregate data metaanalysis involving 9,000 patients with CAD and 8,608 controls from a total of 55 studies, and concluded that CMV infection was associated with an increased risk of CAD (24). In this regard, some suggested potential mechanisms include vessel wall colonization, enhancement of body's pre-existing inflammatory response to risk factors such as hyperlipidemia, destabilization of the pre-existing plaques by activating the immune response, endothelial dysfunction, or even cross-reaction of the anti-CMV antibodies with heat shock proteins during the CMV infection and the subsequent apoptosis of the non-stressed endothelial cells $(16,25-28)$. Yet, the most accepted mechanism through which CMV may contribute to atherogenesis is provocation of inflammatory response in the host (29). The previous studies indicated that CMV was detected in atherosclerotic plaques (30). Moreover, Liu et al. showed that the presence and severity of CMV infection in coronary plaques were associated with the incidence of Acute Coronary Syndrome (ACS) (31).

\section{Objectives}

Considering the controversy that still exists in the field, the present study aims to investigate the seroprevalence of anti-CMV antibodies and inflammatory markers in patients with stable angina who had undergone diagnostic coronary angiography for clinical suspicion of CAD.

\section{Patients and Methods}

This descriptive, cross-sectional study was conducted with the approval of the Institutional and Ethics Review Boards of Urmia University of Medical Sciences, Iran. The patients with stable angina who were referred by a cardiologist for diagnostic coronary angiography to Seyyedoshohada Heart Hospital of Urmia, West-Azerbaijan province, Iran between August 2012 and December 2013 were selected using simple random sampling. However, the patients with myocardial infarction during the last 3 months, those with known malignant diseases or a febrile infection within the last month, the patients who refused to give consent, and those with an unstable condition were excluded from the study. A patient was defined to have CAD if there was an angiographic evidence of any plaques in any segment of the epicardial coronary arteries. On the other hand, a patient was defined as "non-CAD" if all the coronary arteries were judged based on the angiography to be free of plaques.

Prior to angiography, five $\mathrm{mL}$ of arterial blood was obtained from each patient and after being centrifuged, the serum was stored at $-80^{\circ} \mathrm{C}$. The blood samples were tested for anti-CMV IgG and IgM antibodies using the Enzyme-Linked Immunosorbent Assay (ELISA) method (Diapron, Rome, Italy). According to the manufacturer's instructions, $\mathrm{CMV} \mathrm{IgG}>0.5 \mathrm{IU} / \mathrm{mL}$ was considered to be positive, IgM $<8$ Arbitrary Units (AU) $/ \mathrm{mL}$ was determined to be seronegative, $\operatorname{IgM} \geq 8$ but $<12 \mathrm{AU} / \mathrm{mL}$ was considered to be equivocal, and $\operatorname{IgM} \geq 12 \mathrm{AU} / \mathrm{mL}$ was determined as seropositive. It should be noted that positive CMV IgG and IgM in a symptomatic individual implies that one most likely has either an active primary or a reactivated latent CMV infection. Besides, positive CMV IgM and negative IgG means that the individual may have been infected very recently. Positive CMV IgG but negative IgM also reflect past exposure or latent infection. Finally, patients with no current or prior infection are expected to be seronegative for both CMV IgM and IgG assays. In this study, serum C-Reactive Protein (CRP), as a marker of inflammation, was measured by a qualitative method using the commerciallyavailable Aniston Kit. Additionally, information regarding age, sex, fasting blood sugar levels, and lipid profile was gathered through a chart review.

This study was performed on 160 participants with stable angina who underwent diagnostic coronary angiography, with 112 subjects being allocated to the CAD group and 48 ones being allocated to the control group. This sample size was selected based on the prevalence rates of $90 \%$ and $70 \%$ in the CAD and control groups, respectively (32), power of $80 \%$, and two-tailed $\alpha$ of 0.05 (33). The continuous variables were presented as mean \pm Standard Deviation (SD), while the categorical ones were presented as frequency and percentage. The groups with and without CAD were compared using independent $t$-test for continuous variables and Chi-square or Fisher's exact test for categorical variables. Given the high seroprevalence of anti-CMV antibodies in both groups, no multivariate regression analysis was performed. All the statistical analyses were done using the IBM SPSS statistical software, version 21 and $\mathrm{P}<0.05$ was considered to be statistically significant.

\section{Results}

This cross-sectional study was conducted on 181 patients who were referred by a cardiologist for diagnostic coronary angiography. The mean age of the patients was $57.6 \pm 11.1$ years (age range: 29 - 95 years) and $87.1 \%$ were 45 years of age and above. Additionally, 91 cases (50.3\%) were male, $41.6 \%$ suffered from diabetes, and $70.6 \%$ had dyslipidemia.

Coronary plaques were observed in a single coronary artery in 77 (42.5\%), in two coronary arteries in $29(16.0 \%)$, and in all the three major coronary branches (including left anterior descending, left circumflex, and right coronary arteries) in 35 patients (19.3\%). On the other hand, coronary angiography was free of any plaques in forty cases (22.1\%) that were considered to be "non-CAD".

Based on the results of ELISA, 171 (99.4\%) and 21 (12.0\%) cases were seropositive for anti-CMV IgG and IgM, respectively. Also, 112 cases (62.9\%) were seropositive for CRP. The results of comparison of the two groups regarding the patients' characteristics and laboratory test results have been presented in Tables 1 and 2. Accordingly, the patients' mean age was higher in the CAD group compared to the non-CAD group $(58.7 \pm 10.9$ vs. $53.6 \pm 10.9 ; \mathrm{P}=0.01)$. However, this age difference did not remain significant after categorization of the patients to ages $<45$ years and 45 years 


\begin{tabular}{|c|c|c|c|c|}
\hline & Total $(\mathrm{N}=181)$ & CAD Group $(\mathrm{N}=141)$ & Non-CAD Group $(\mathrm{N}=40)$ & P value \\
\hline \multicolumn{5}{|l|}{ Characteristics } \\
\hline Age & $57.6 \pm 11.1$ & $5.8 \pm 10.9$ & $53.6 \pm 10.9$ & 0.011 \\
\hline Age $\geq 45$ years; $n(\%)$ & $155(87.1 \%)$ & $124(88.6 \%)$ & $31(81.6 \%)$ & 0.278 \\
\hline Male Sex; n (\%) & $91(50.3 \%)$ & $76(53.9 \%)$ & $15(37.5 \%)$ & 0.067 \\
\hline Dyslipidemia; n (\%) & $60.85(70.6 \%)$ & $49.65(75.4 \%)$ & $11.20(55.0 \%)$ & 0.080 \\
\hline \multicolumn{5}{|l|}{ Test results } \\
\hline Total Cholesterol (mg/dL) & $179.1 \pm 50.5$ & $183.5 \pm 52.9$ & $164.5 \pm 39.2$ & 0.152 \\
\hline HDL (mg/dL) & $42.6 \pm 11.1$ & $42.3 \pm 10.1$ & $43.8 \pm 14.0$ & 0.602 \\
\hline LDL (mg/dL) & $93.1 \pm 31.1$ & $96.2 \pm 32.8$ & $83.5 \pm 23.0$ & 0.110 \\
\hline Triglyceride (mg/dL) & $166.8 \pm 89.6$ & $167.7 \pm 90.3$ & $164.0 \pm 89.5$ & 0.871 \\
\hline FBS & $131.2 \pm 56.8$ & $137.6 \pm 61.1$ & $105.2 \pm 20.2$ & $<0.001$ \\
\hline $\mathrm{FBS} \geq 126 \mathrm{mg} / \mathrm{dL} ; \mathrm{n}(\%)$ & $47(38.8 \%)$ & $40(41.2 \%)$ & $7(29.2 \%)$ & 0.277 \\
\hline
\end{tabular}

Abbreviations: CAD, coronary artery disease; FBS, fasting blood sugar; HDL, high-density lipoprotein; LDL: low-density lipoprotein

\begin{tabular}{llll}
\hline Table 2. The Main Study Findings & & & \\
\hline & CAD Group (N = 141) & Non-CAD Group (N = 40) & P value \\
\hline Anti-CMV lgG Seropositivity; $\mathbf{n}(\%)$ & $134.135(99.3 \%)$ & $37.37(100 \%)$ & 1.000 \\
Anti-CMV lgM Seropositivity; $\mathbf{n}(\%)$ & $16.137(11.7 \%)$ & $5.38(13.2 \%)$ & 0.782 \\
Postitive CRP; $\mathbf{n}(\%)$ & $90.139(64.7 \%)$ & $22.39(56.4 \%)$ & 0.341 \\
\hline
\end{tabular}

Abbreviations: CAD, Coronary artery disease; CMV, Cytomegalovirus; CRP, C-reactive protein

or above $(\mathrm{P}=0.27)$. Also, no significant sex difference was found between the groups with and without $\mathrm{CAD}(\mathrm{P}=0.06)$. Moreover, almost all the cases (99.3 in the CAD group and $100 \%$ in the non-CAD group) were anti-CMV IgG positive. As expected, the rates were lower for anti-CMV IgM $(11.7 \%$ in the CAD group versus $13.2 \%$ in the non-CAD group, $\mathrm{P}=$ $0.78)$. Nonetheless, no significant difference was observed between the groups with and without angiographicallydocumented CAD in terms of CRP seropositivity ( $\mathrm{P}=$ 0.34). Regardless of the CAD classification, the rate of CRP seropositivity was significantly higher in the CMV IgM positive group compared to the IgM negative patients $(95.2 \%$ vs. $52.8 \%, \mathrm{P}=0.001)$.

\section{Discussion}

This study was conducted on 181 patients who underwent diagnostic coronary angiography for clinical suspicion of CAD. According to the study findings, the rate of antiCMV IgG seropositivity was very high and almost all the patients had previous exposure to CMV infection. Besides, no significant difference was found between the groups with and without CAD in terms of their previous exposure to CMV infection. Also, no significant association was observed between CRP (as the marker of inflammation) and CAD.

Another study by our group showed an anti-CMV IgG seropositivity rate of $77.4 \%$ among hemodialysis patients (2), which is lower than the rate observed among the CAD patients in the current study. This observation is actually intriguing considering the compromised immune function in patients with end-stage renal disease. On one hand, immunodeficiency predisposes patients to viral and bacterial infections and, on the other hand, the immune system is unable to produce an adequate amount of antibody in immunocompromised patients. The high rate of anti-CMV IgG seropositivity is not a rare phenomenon even in healthy individuals. A study in northwest of Iran revealed more than $98 \%$ seropositivity for CMV in a group of healthy blood donors (34). Also, the results of the research by Nikolopoulou et al. indicated the rate of anti-CMV IgG to be $95.5 \%$ among the patients with angiographically-documented stable CAD (35), which is similar to our findings in the CAD group. Alavi et al. also performed a study in southern Iran and reported a high rate of anti-CMV IgG seropositivity in patients with CAD as well as in controls $(93 \%$ of the patients with unstable angina and $96.7 \%$ of the controls) (11).

In a similar study by Safaie et al. in Iran's East Azerbaijan province (a neighboring province to ours), CMV infection was detected in $83 \%$ of the CAD cases and $63 \%$ of the non-CAD group. That specific study showed a significant association between CMV infection and CAD (32). However, because of the very high rate of CMV IgG seropositivity, we failed to evaluate any association between CMV infection and CAD in the current study.

Although many previous studies showed a significantly higher rate of CRP seropositivity in individuals with CAD compared to those without $(5,10,17)$, we failed to find any significant associations between CRP seropositivity and the presence of CAD. One limitation could be the use of contemporary CRP assays instead of high-sensitive CRP test in this study. It could also result from the possible difference between the non-CAD groups in different studies. In our study, the non-CAD group included the patients who were referred by a cardiologist for diagnostic coronary angiography and their coronary trees were free of plaques. Naturally, there was some suspicion of CAD in these patients, which made them a candidate for coronary angiography. Hence, these patients might have some spectrums of atherosclerosis, but have not yet established a disease. Especially in Urmia, West-Azerbaijan province, because of the small number of interventionists and 
limited resources, cardiologists have to be very selective on referring patients for diagnostic coronary angiography.

In the present study, ELISA method was used to detect the CMV-specific IgG antibodies in the serum. One of the suggested mechanisms for the role of CMV in atherogenesis is CMV colonization at the vessel wall cells. It should be noted that infection in the vessel wall may not be detected by antibodies in the serum (36). Based on the meta- analysis performed by Ji et al., only seven studies so far have used the Polymerase Chain Reaction (PCR) assay, as a more sensitive test, for detecting CMV infection. According to the results of this meta-analysis, these PCR studies revealed a much stronger association between CMV infection and CAD compared to ELISA studies $(\mathrm{OR}=8.12$ for PCR studies vs. 1.56 for ELISA studies) (24).

It should be mentioned that acute, sub-acute, and chronic infections may play differential roles in inducing atherosclerosis and the subsequent cardiovascular diseases. To date, chronic infection has been proposed to be the main culprit for the role of CMV in atherogenesis. Yet, further studies are required to be conducted in this area. Although we had data about IgM seropositivity in the current study, the criterion for chronic infection was defined as detection of anti-CMV IgG antibodies in the serum. Thus, the CMVspecific IgG data were of more use here compared to the anti-CMV IgM data.

In conclusion, regardless of having angiographicallyproven CAD, almost all the cases who referred for diagnostic coronary angiography in our study had a previous exposure to CMV infection as determined by the presence of anti-CMV IgG antibodies in their sera. In fact, no significant association was observed between CMV infection and the presence of CAD.

\section{Acknowledgements}

The authors would like to thank Urmia University of Medical Sciences for providing the grant for the study.

\section{Authors' Contribution}

Zakieh Rostamzadeh Khameneh was the principal investigator of this project. All the co-authors contributed to study design. Mohaddeseh Nemati did the serological tests. Paul Brown did the analysis. Nariman Sepehrvand contributed to writing of the manuscript and all the coauthors approved the final version for publication.

\section{Financial disclosure}

There is no financial disclosure.

\section{Funding/Support}

This study was supported by Urmia University of Medical Sciences.

\section{References}

1. Khameneh ZR, Sepehrvand N, Aghazadeh T. Cytomegalovirus infection among Iranian kidney graft recipients. Transplant Proc. 2013;45(1):178-81.

2. Sepehrvand N, Khameneh ZR, Eslamloo HR. Survey the seroprevalence of CMV among hemodialysis patients in Urmia, Iran. Saudi J Kidney Dis Transpl. 2010;21(2):363-7.

3. Eryol NK, Kilic H, Gul A, Ozdogru I, Inanc T, Dogan A, et al. Are the high levels of cytomegalovirus antibodies a determinant in the development of coronary artery disease? Int Heart $J$. 2005;46(2):205-9.

4. Zhou YF, Shou M, Guetta E, Guzman R, Unger EF, Yu ZX, et al. Cytomegalovirus infection of rats increases the neointimal response to vascular injury without consistent evidence of direct infection of the vascular wall. Circulation. 1999;100(14):1569-75.

5. Anderson JL, Carlquist JF, Muhlestein JB, Horne BD, Elmer SP. Evaluation of C-reactive protein, an inflammatory marker, and infectious serology as risk factors for coronary artery disease and myocardial infarction. J Am Coll Cardiol. 1998;32(1):35-41.

6. Danesh J, Wong Y, Ward M, Muir J. Chronic infection with Helicobacter pylori, Chlamydia pneumoniae, or cytomegalovirus: population based study of coronary heart disease. Heart. 1999;81(3):245-7.

7. Fang JC, Kinlay S, Kundsin R, Ganz P. Chlamydia pneumoniae infection is frequent but not associated with coronary arteriosclerosis in cardiac transplant recipients. Am J Cardiol. 1998;82(12):1479-83.

8. Georges JL, Rupprecht HJ, Blankenberg S, Poirier O, Bickel C, Hafner $\mathrm{G}$, et al. Impact of pathogen burden in patients with coronary artery disease in relation to systemic inflammation and variation in genes encoding cytokines. Am J Cardiol. 2003;92(5):515-21.

9. Gredmark S, Jonasson L, Van Gosliga D, Ernerudh J, SoderbergNaucler C. Active cytomegalovirus replication in patients with coronary disease. Scand Cardiovasc J. 2007;41(4):230-4.

10. Jha HC, Prasad J, Mittal A. High immunoglobulin A seropositivity for combined Chlamydia pneumoniae, Helicobacter pylori infection, and high-sensitivity $\mathrm{C}$-reactive protein in coronary artery disease patients in India can serve as atherosclerotic marker. Heart Vessels. 2008;23(6):390-6.

11. Alavi SM, Adel SM, Rajabzadeh AR. An evidence against the effect of chronic cytomegalovirus infection in unstable angina pectoris. Acta Med Iran. 2011;49(2):78-80.

12. Altannavch T, Roubalova K, Broz J, Hruba D, Andel M. Serological markers of Chlamydia pneumoniae, cytomegalovirus and Helicobacter pylori infection in diabetic and non-diabetic patients with unstable angina pectoris. Cent Eur J Public Health. 2003;11(2):102-6.

13. Ammann P, Marschall S, Kraus M, Schmid L, Angehrn W, Krapf $\mathrm{R}$, et al. Characteristics and prognosis of myocardial infarction in patients with normal coronary arteries. Chest. 2000;117(2):333-8.

14. Arcari CM, Gaydos CA, Nieto FJ, Krauss M, Nelson KE. Association between Chlamydia pneumoniae and acute myocardial infarction in young men in the United States military: the importance of timing of exposure measurement. Clin Infect Dis. 2005;40(8):1123-30.

15. Bloemenkamp DG, Mali WP, Tanis BC, Rosendaal FR, van den Bosch MA, Kemmeren JM, et al. Chlamydia pneumoniae, Helicobacter pylori and cytomegalovirus infections and the risk of peripheral arterial disease in young women. Atherosclerosis. 2002;163(1):149-56.

16. Chen R, Xiong S, Yang Y, Fu W, Wang Y, Ge J. The relationship between human cytomegalovirus infection and atherosclerosis development. Mol Cell Biochem. 2003;249(1-2):91-6.

17. De Backer J, Mak R, De Bacquer D, Van Renterghem L, Verbraekel E, Kornitzer M, et al. Parameters of inflammation and infection in a community based case-control study of coronary heart disease. Atherosclerosis. 2002;160(2):457-63.

18. Hoffmeister A, Rothenbacher D, Bode G, Persson K, Marz W, Nauck MA, et al. Current infection with Helicobacter pylori, but not seropositivity to Chlamydia pneumoniae or cytomegalovirus, is associated with an atherogenic, modified lipid profile. Arterioscler Thromb Vasc Biol. 2001;21(3):427-32.

19. Rothenbacher D, Brenner H, Mertens T, Hoffmann MM, Hoffmeister A, Koenig W. Prognostic value of interleukin-1 receptor antagonist gene polymorphism and cytomegalovirus seroprevalence in patients with coronary artery disease. BMC Cardiovasc Disord. 2005;5(1):10.

20. Sheehan J, Kearney PM, Sullivan SO, Mongan C, Kelly E, Perry IJ. Acute coronary syndrome and chronic infection in the Cork coronary care case-control study. Heart. 2005;91(1):19-22.

21. Smieja M, Cronin L, Levine M, Goldsmith CH, Yusuf S, Mahony JB. Previous exposure to Chlamydia pneumoniae, Helicobacter pylori and other infections in Canadian patients with ischemic heart disease. Can J Cardiol. 2001;17(3):270-6.

22. Sun Y, Pei W, Welte T, Wu Y, Ye S, Yang Y. Cytomegalovirus infection is associated with elevated interleukin-10 in coronary artery disease. Atherosclerosis. 2005;179(1):133-7. 
23. Witherell HL, Smith KL, Friedman GD, Ley C, Thom DH, Orentreich N, et al. C-reactive protein, Helicobacter pylori, Chlamydia pneumoniae, cytomegalovirus and risk for myocardial infarction. Ann Epidemiol. 2003;13(3):170-7.

24. Ji YN, An L, Zhan P, Chen XH. Cytomegalovirus infection and coronary heart disease risk: a meta-analysis. Mol Biol Rep. 2012;39(6):6537-46.

25. Bason C, Corrocher R, Lunardi C, Puccetti P, Olivieri O, Girelli $\mathrm{D}$, et al. Interaction of antibodies against cytomegalovirus with heat-shock protein 60 in pathogenesis of atherosclerosis. Lancet. 2003;362(9400):1971-7.

26. Grahame-Clarke C, Chan NN, Andrew D, Ridgway GL, Betteridge DJ, Emery V, et al. Human cytomegalovirus seropositivity is associated with impaired vascular function. Circulation. 2003;108(6):678-83.

27. Muhlestein JB, Anderson JL. Chronic infection and coronary artery disease. Cardiol Clin. 2003;21(3):333-62.

28. Weis M, Kledal TN, Lin KY, Panchal SN, Gao SZ, Valantine HA, et al. Cytomegalovirus infection impairs the nitric oxide synthase pathway: role of asymmetric dimethylarginine in transplant arteriosclerosis. Circulation. 2004;109(4):500-5.

29. Zhu J, Quyyumi AA, Norman JE, Csako G, Epstein SE. Cytomegalovirus in the pathogenesis of atherosclerosis: the role of inflammation as reflected by elevated C-reactive protein levels. J Am Coll Cardiol. 1999;34(6):1738-43.
30. Paoletti R, Gotto AM, Jr., Hajjar DP. Inflammation in atherosclerosis and implications for therapy. Circulation. 2004;109(23 Suppl 1):III20-6.

31. Liu R, Moroi M, Yamamoto M, Kubota T, Ono T, Funatsu A, et al. Presence and severity of Chlamydia pneumoniae and Cytomegalovirus infection in coronary plaques are associated with acute coronary syndromes. Int Heart J. 2006;47(4):511-9.

32. Safaie N, Ghotaslou R, Montazer Ghaem H. Seroprevalence of cytomegalovirus in patients with and without coronary artery diseases at Madani Heart Center, Iran. Acta Med Iran. 2010;48(6):403-6.

33. Hulley SB, Cummings SR, Browner WS, Grady DG, Newman TB. Designing clinical research. Lippincott Williams \& Wilkins; 2013.

34. Eivazi-Ziaei J, Movassagpour A, Asgharzadeh M, Dastgiri S. Seroprevalence of cytomegalovirus in blood donors in the northwest of Iran. JArCM. 2013;1(2):96-100.

35. Nikolopoulou A, Tousoulis D, Antoniades C, Petroheilou K, Vasiliadou C, Papageorgiou N, et al. Common community infections and the risk for coronary artery disease and acute myocardial infarction: evidence for chronic over-expression of tumor necrosis factor alpha and vascular cells adhesion molecule-1. Int J Cardiol. 2008;130(2):246-50.

36. Tornvall P, Saleh N, Zweygberg-Wirgart B, Grillner L. Cytomegalovirus in coronary heart disease. Atherosclerosis. 2006;184(1):235. 